\title{
Modeling Health Diseases Using Competitive Fuzzy Cognitive Maps
}

\author{
Antigoni P. Anninou ${ }^{1}$, Peter P. Groumpos ${ }^{1}$, and Panagiotis Polychronopoulos ${ }^{2}$ \\ ${ }^{1}$ Laboratory for Automation and Robotics, Department of Electrical and \\ Computer Engineering, University of Patras, Greece \\ \{anninou, groumpos\} @ece.upatras.gr \\ ${ }^{2}$ Clinic of Neurology, Department of Pathology, General University Hospital of Patras \\ ppolychr@yahoo.gr
}

\begin{abstract}
This paper presents the medical decision support systems (MDSS) and their architecture. The aim of this paper is to present a new approach in modeling knee injuries using Competitive Fuzzy Cognitive Maps (CFCMs). Basic theories of CFCMs are reviewed and presented. Decision Support Systems (DSS) for Medical problems are considered. Finally, it illustrates the development of an MDSS for finding knee injury with the architecture of CFCMs.
\end{abstract}

Keywords: Fuzzy cognitive maps, medical decision support system, competitive fuzzy cognitive maps, knee injuries.

\section{Introduction}

A fuzzy cognitive map (FCM) is a soft computing technique, which is capable of dealing with complex systems in situations exactly as a human does using a reasoning process that can include uncertain and ambiguity descriptions [6], [9]. FCM is a promising modeling method for describing particular domains showing the concepts (variables) and the relationships between them (weights) while it encompasses advantageous features [8]. Fuzzy Cognitive Maps (FCM) are fuzzy-graph structures for representing causal reasoning. Their fuzziness allows hazy degrees of causality between causal objects (concepts) [2].

In this paper a decision support system will be implemented. Decision Support System (DSS) is defined as any interactive computer - based support system for making decisions in any complex system, when individuals or a team of people are trying to solve unstructured problems on an uncertain environment. Medical Decision Systems have to consider a high amount of data and information from interdisciplinary sources (patient's records and information, doctors' physical examination and evaluation, laboratory tests, imaging tests) and, in addition to this, medical information may be vague, or missing [1-2]. Furthermore the Medical Diagnosis procedure is complex, taking into consideration a variety of inputs in order to infer the final diagnosis. Medical Decision Systems are complex ones, consisting of 
irrelevant and relevant subsystems and elements, taking into consideration many factors that may be complementary, contradictory and competitive; these factors influence each other and determine the overall diagnosis with a different degree. It is apparent that these systems require a modeling tool that can handle all these challenges and at the same time to be able to infer a decision. Thus, FCMs are suitable to model a Medical Decision Support Systems (MDSS) [2].

A special type of FCM has been introduced for Medical Diagnosis systems, with advanced capabilities, the Competitive Fuzzy Cognitive Map (CFCM) [4-5]. Each decision concept represents a single decision, which means that these concepts must compete against each other so that only one of them dominates and is considered as the correct decision. This is the case of most medical applications, where, the symptoms have to conclude to one diagnosis. The factor-concepts take values from patient data. These concepts represent symptoms, experimental and laboratory tests, and their values are dynamically updated. The decision concepts are considered as outputs in which their calculated values outline the possible diagnosis for the patient [3].

In this paper we present in a simple but illustrative way how useful the FCMs can be in medical problems. In section 2 the basic theories of CFCM, while the decision making support system in knee injuries is described in Section 3. The paper concludes in section 4.

\section{Competitive Fuzzy Cognitive Maps}

The algorithm that describes the Competitive Fuzzy Cognitive Maps consists of the following steps [3]:

Step 1: Set values $A_{i}$ of nodes according to values of the factors involved in the decision process. The values $\mathrm{Ai}$ are described using linguistic variables.

Step 2: Read the weight matrix W. The interrelationships between nodes may be positive, negative or zero. The existing relationships between the concepts are described firstly, as "negative" or "positive" and secondly, as a degree of influence using a linguistic variable, such as "low", "medium", "high", etc. A positive weight means that the specific factor increases the possibility of diagnosis the interconnected disease. A negative one reduces the possibility of diagnosis the interconnected disease. Lack of interconnection indicates that there is no impact of the factor to the disease. By using a defuzzification method [10], weights between the factor-concepts and the decision-concepts are converted to initial values, which for the current research are between 0 and 1. These are placed in matrix $\mathrm{W}$ of size $(n+m)$ $\mathrm{x}(n+m)$. The values in the first $n$ columns correspond to the weighted connections from all the concepts towards the $n$ decision-concepts, and the values in the remaining $m$ columns correspond to the weighted connections from all the concepts towards the factor-concepts. This matrix also includes the -1 weight values for competition between output decision-concepts.

Step 3: Update the values: $\mathrm{A}_{\text {new }}=\mathrm{A}_{\text {old }} * \mathrm{~W}$. 
Step 4: $A=f\left(A_{\text {new }}\right)$, where the sigmoid function $f$ belongs to the family of squeezing functions, and the following function is usually used to describe it:

$$
f=\frac{1}{1+e^{-\lambda x}}
$$

This is the unipolar sigmoid function, in which $\lambda>0$ determines the steepness of the continuous function $\mathrm{f}(\mathrm{x})$.

Step 5: Repeat steps until equilibrium has been reached and the values of the concepts no longer change.

Step 6: The procedure stops and the final values of the decision-concepts are found, the maximum of which is the chosen decision.

Although the model FCMs-MDSS designed to include all possible symptoms, the causal factors and their relationships, there is a particular situation in which very few of the symptoms are available and taken into account. Thus, in such a diagnostic or prognostic model FCMs-MDSS, the decision will be based using only a small part of the nodes of the entire system, leading to a wrong decision or difficulty convergence since weights of active nodes reflect a small part of the knowledge of specialists.

By using a FCM augmented with a Case-Based-Reasoning (CBR), in such situations, the DSS would draw upon cases that are similar according to distance measures and would use the CBR subsystem to generate a sub-FCM emphasizing the nodes activated by the patient data and thus redistributing the causal weights between the concept-nodes.

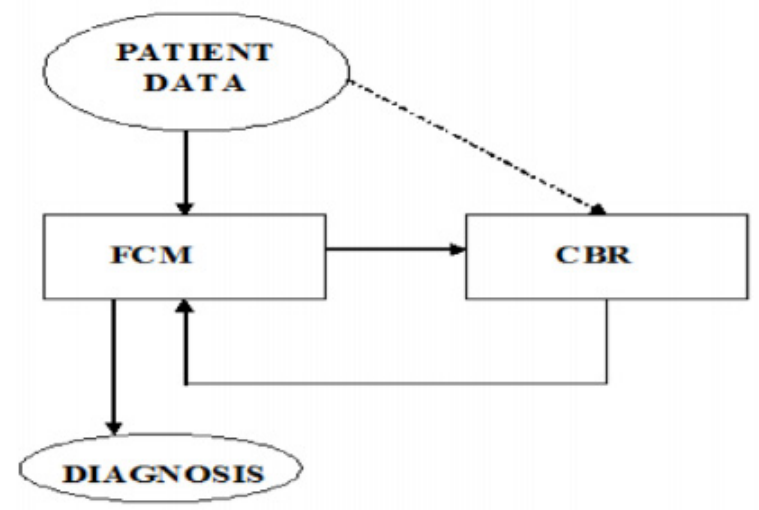

Fig. 1. FCM-CBR

\section{Competitive Fuzzy Cognitive Maps for Knee Injuries}

Knee pain is a common complaint for many people. There are several factors that can cause knee pain. Awareness and knowledge of the causes of knee pain lead to a more 
accurate diagnosis. Management of knee pain is in the accurate diagnosis and effective treatment for that diagnosis. Knee pain can be either referred pain or related to the knee joint itself [7].

CFCM distinguish two main types of nodes: decision-nodes (decision-concepts) and factor-nodes [5]. The following figure presents an example model CFC $\mathrm{M}$ that is used in order to determine the medical diagnosis, and includes types of FCMs concepts and causal relationships between them [3].

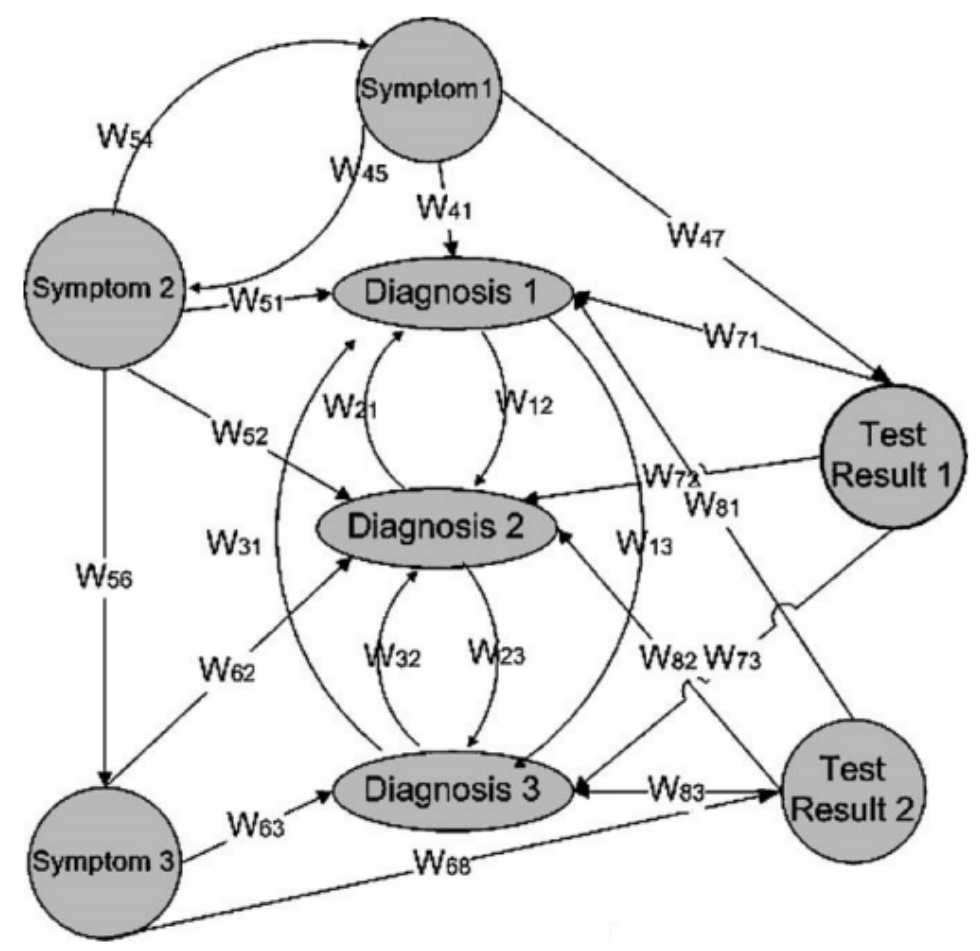

Fig. 2. A General Medical Decision Support CFCM

All concepts may interact with each other and decide the value of diagnosis-nodes that are mutually exclusive in order to show one single diagnosis. In most medical applications according to symptoms, physicians need to complete only one diagnosis and then to determine the appropriate treatment.

In the specific model that describes knee injuries, there are two kinds of nodes:

- $\mathrm{n}$ decision-nodes (as outputs):

$\mathbf{C}_{\mathbf{1}}$ : Overuse injuries

$\mathbf{C}_{2}$ : Patellofemoral pain syndrome

$\mathbf{C}_{3}$ : Osteochondritis dissecans of the femoral condyles

$\mathbf{C}_{4}$ : Tibial tuberosity apophysitis 
$\mathbf{C}_{5}$ : Iliotibial band syndrome

$\mathbf{C}_{6}$ : Biceps femoris tendinitis

$\mathbf{C}_{7}$ : Inferior patellar pole osteochondritis

$-\mathrm{m}$ factor-nodes (as inputs):
$\mathbf{C}_{\mathbf{8}}$ : Patellar Instability
$\mathbf{C}_{9}$ : Patellar Crepitation
$\mathbf{C}_{\mathbf{1 0}}$ : Patella Dislocation
$\mathbf{C}_{11}$ : Patella Pain
$\mathbf{C}_{12}$ : Rectus Femoris Pain
$\mathbf{C}_{13}$ : Muscle Pain
$\mathbf{C}_{14}$ : Quadriceps Pain
$\mathbf{C}_{15}$ : Tendon Injury
$\mathbf{C}_{\mathbf{1 6}}$ : Edema
$\mathbf{C}_{17}$ : Heat
$\mathbf{C}_{\mathbf{1 8}}$ : Passive Hyperextension Pain
$\mathbf{C}_{\mathbf{1 9}}$ : Active Hyperextension Pain
$\mathbf{C}_{\mathbf{2 0}}$ : Pain during exercise
$\mathbf{C}_{21}$ : Patellofemoral Joint Crepitation

Fig. 3 shows the representation of the 15 factor-nodes (C8-C21), 7 decision-nodes (C1-C7) and the relationships between them.

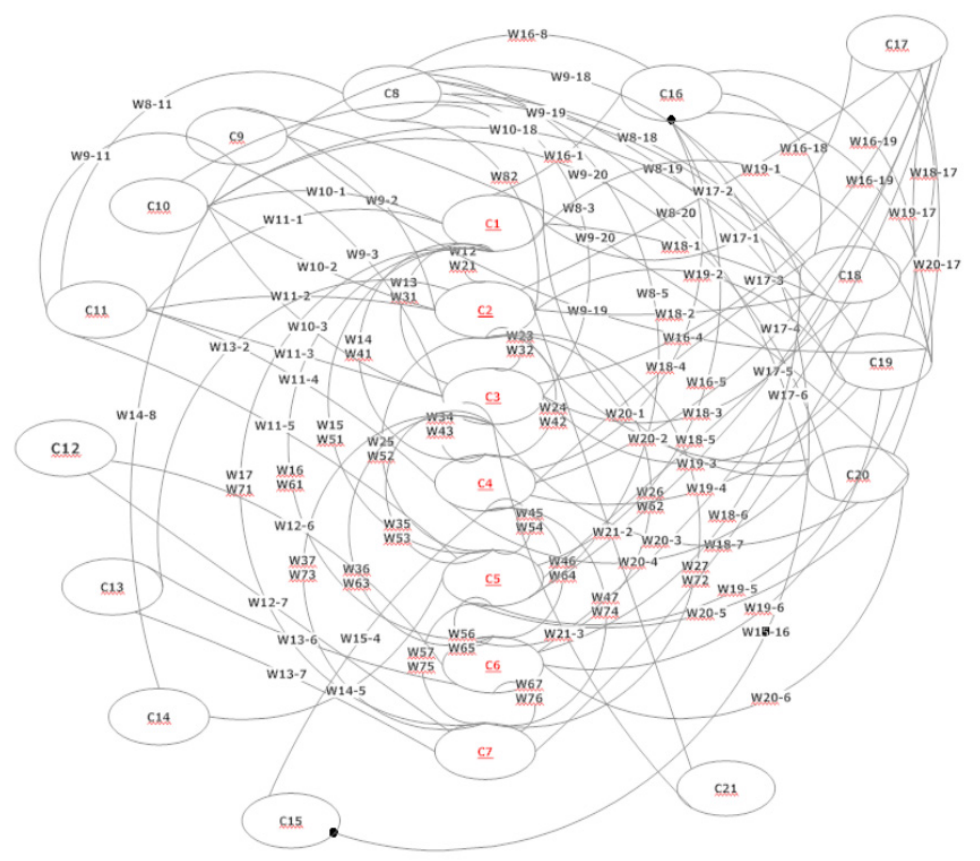

Fig. 3. Competitive Fuzzy Cognitive Map for Knee Injuries 
According to the above algorithm and the kind of injuries, the weight matrix $\mathrm{W}$ would be as follows using linguistic variables:

Table 1. Linguistic Variables of the Concepts Relationships

\begin{tabular}{|c|c|c|c|c|c|c|c|c|c|c|c|c|c|c|c|c|c|c|c|c|c|}
\hline & C1 & C2 & C3 & C4 & C5 & C6 & C7 & $c 8$ & C9 & C10 & C11 & C12 & C13 & C14 & C15 & C16 & C17 & C18 & C19 & $C_{2} 0$ & $\mathrm{C} 21$ \\
\hline C1 & & -1 & -1 & -1 & -1 & -1 & -1 & & & & & & & & & & & & & & \\
\hline C2 & -1 & & -1 & -1 & -1 & -1 & -1 & & & & & & & & & & & & & & \\
\hline C3 & -1 & -1 & & -1 & -1 & -1 & -1 & & & & & & & & & & & & & & \\
\hline C4 & -1 & -1 & -1 & & -1 & -1 & -1 & & & & & & & & & & & & & & \\
\hline C5 & -1 & -1 & -1 & -1 & & -1 & -1 & & & & & & & & & & & & & & \\
\hline C6 & -1 & -1 & -1 & -1 & -1 & & -1 & & & & & & & & & & & & & & \\
\hline C7 & -1 & -1 & -1 & -1 & -1 & -1 & & & & & & & & & & & & & & & \\
\hline C8 & $+\mathrm{M}$ & $+\mathrm{VH}$ & $+V V H$ & None & $+V V L$ & None & None & & & & $+\mathrm{L}$ & & & & & & & $+\mathrm{L}$ & $+\mathrm{L}$ & $+\mathrm{L}$ & \\
\hline C9 & None & $+V L$ & $+\mathrm{VH}$ & None & None & None & None & & & & $+\mathrm{L}$ & & & & & & & $+L$ & $+\mathrm{L}$ & th & \\
\hline C10 & $+\mathrm{M}$ & +VVL & $+\mathrm{VH}$ & None & None & None & None & & & & $+L$ & & & & & & & $+L$ & $+L$ & $+\mathrm{L}$ & \\
\hline C11 & $+\mathrm{VVH}$ & $+\mathrm{VH}$ & H & $+V V H$ & $+M$ & None & None & & & & & & & & & & & & & & \\
\hline C12 & None & None & None & None & None & $+\mathrm{VH}$ & $+\mathrm{VVH}$ & & & & & & & & & & & & & & \\
\hline C13 & None & $+M$ & None & None & None & $+V V H$ & $+\mathrm{VH}$ & & & & & & & & & & & & & & \\
\hline C14 & None & None & None & None & $+\mathrm{VVH}$ & None & None & $+\mathrm{L}$ & & & & & & & & & & & & & \\
\hline C15 & None & None & None & $+V V H$ & None & None & None & & & & & & & & & $+\mathrm{L}$ & & & & & \\
\hline C16 & $+\mathrm{H}$ & None & None & $+\mathrm{VH}$ & $+\mathrm{VVH}$ & None & None & $+\mathrm{L}$ & & & & & & & & & & $+L$ & $+\mathrm{L}$ & $+L$ & \\
\hline C17 & $+\mathrm{H}$ & $+M$ & $+M$ & $+M$ & $+\mathrm{VVH}$ & $+V L$ & None & & & & & & & & & & & & & & \\
\hline C18 & $+L$ & $+H$ & $+H$ & $+\mathrm{VH}$ & $+L$ & $+\mathrm{M}$ & $+\mathrm{VH}$ & & & & & & & & & & $+L$ & & & & \\
\hline C19 & $+L$ & $+\mathrm{VH}$ & $+H$ & $+\mathrm{VVH}$ & $+M$ & $+\mathrm{M}$ & $+L$ & & & & & & & & & & $+L$ & & & & \\
\hline $\mathrm{C} 20$ & $+\mathrm{VH}$ & $+V V H$ & $+\mathrm{VH}$ & $+\mathrm{VH}$ & $+M$ & $+\mathrm{H}$ & $+L$ & & & & & & & & & & $+L$ & & & & \\
\hline C21 & None & $+V V L$ & $+M$ & None & None & None & None & & & & & & & & & & & & & & \\
\hline
\end{tabular}

where:

VVH: Very Very High

VH: Very High

H: High

M: Medium

L: Low

VL: Very Low

VVL: Very Very Low

It's obvious that in order to achieve the competition, the relationships between each of these nodes with the rest should have a very large negative weight (-1). This implies that the higher price of a given node, should lead to a reduction in the values of competing nodes.

After defuzzification method Table 1 is converted to the following table:

Table 2. Numerical values of the Concepts Relationships

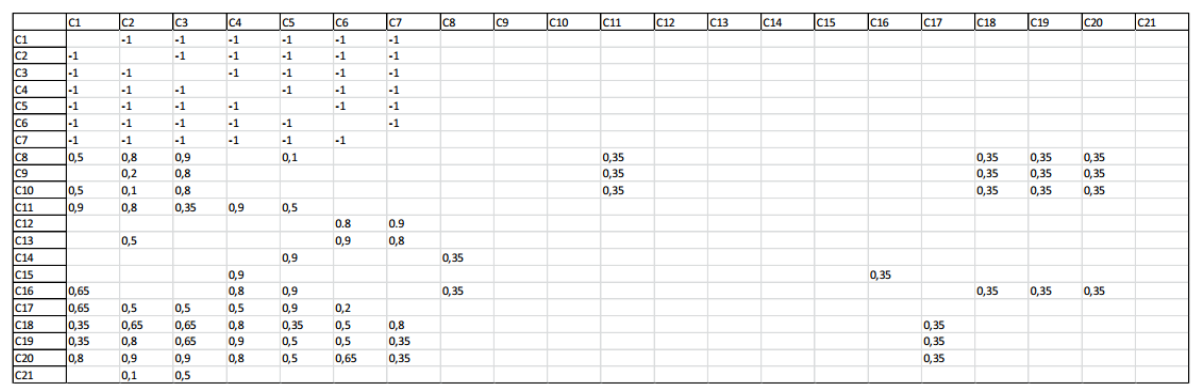


After using the recursive algorithm each node converges to a final value and the decision node with the maximum value is the most possible diagnosis based on the model. In any case, if the model is correct, even with incomplete data, the result will confirm the experts' diagnosis.

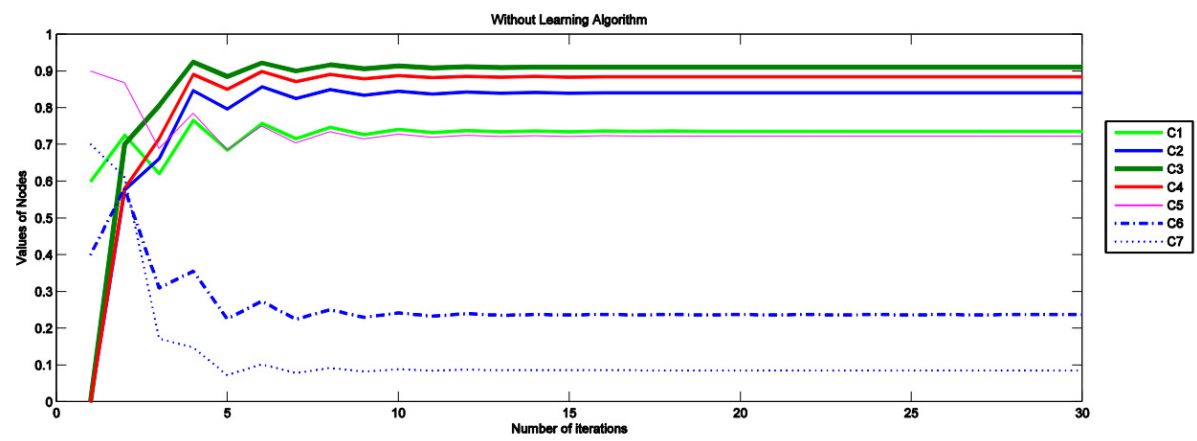

Fig. 4. Subsequent values of concepts till convergence

We observe that in the specific case there is more than one output that has also high convergence values. This means that these types of injuries are very similar because of the adjacent symptoms. So patellofemoral pain syndrome, osteochondritis dissecans of the femoral condyles, and tibial tuberosity apophysitis are the predominant types of injuries that the patient of the example may suffer.

\section{Conclusions}

There is a need for software tools for Medical Decision Support Systems using theories of FCMs. In addition learning algorithms, such as Hebbian and non-linear, should be considered to further investigate Medical problems. The challenging research is the development of new suitable software tools. An interesting research topic is the development of recursive dynamic state equations for FCMs and the use of them for mathematical modeling of medical problems.

This paper deals with a promising future research area. This approach that exploits the experts' knowledge and experience in a modern medical automation system can be used both for diagnosing diseases and also proposing a treatment. More simulation results and more examples are necessary in order to compute the system's reliability in the diagnosis of a specific injury. The model requires further research. To implement this, the cooperation of both physicians and engineers is necessary. The first ones should determine the right concepts and the rules and the second ones are responsible for the implementation of this problem. 


\section{References}

1. Groumpos, P.P., Anninou, A.P.: A theoretical mathematical modeling of Parkinson's disease using Fuzzy Cognitive Maps. In: 12th IEEE International Conference on Bioinformatics and Bioengineering, Cyprus, pp. 677-682 (2012)

2. Anninou, A.P., Groumpos, P.P.: Nonlinear Hebbian Learning Techniques and Fuzzy Cognitive Maps in Modeling the Parkinson's Disease. In: 21st Mediterranean Conference on Control and Automation, Chania, pp. 709-715 (2013)

3. Georgopoulos, V.C., Stylios, C.D.: Competitive Fuzzy Cognitive Maps Combined with Case Based Reasoning for Medical Decision Support. In: Magjarevic, R., Nagel, J.H. (eds.) World Congress on Medical Physics and Biomedical Engineering 2006. IFMBE Proceedings, vol. 14, pp. 3673-3676. Springer, Heidelberg (2006)

4. Georgopoulos, V.C., Stylios, C.D.: Augmented fuzzy cognitive maps supplemented with case based reasoning for advanced medical decision support. In: Nikravesh, M., Zadeh, L.A., Kacprzyk, J. (eds.) Soft Computing for Information Processing and Analysis 2005. STUDFUZZ, vol. 164, pp. 391-405. Springer, Heidelberg (2005)

5. Georgopoulos, V.C., Malandraki, G.A., Stylios, C.D.: A fuzzy cognitive map approach to differential diagnosis of specific language impairment. Artif. Intell. Med. 29(3), 261-278 (2003)

6. Stylios, C.D., Groumpos, P.P.: Modeling complex systems using fuzzy cognitive maps. IEEE Transactions on Systems, Man and Cybernetics, Part A: Systems and Humans 34, $155-162$ (2004)

7. Majewski, M., Susanne, H., Klaus, S.: Epidemiology of athletic knee injuries: A 10-year study. Knee 13(3), 184-188 (2006)

8. Papageorgiou, E.I., Spyridonos, P.P., Glotsos, D.T., Stylios, C.D., Ravazoula, P., Nikiforidis, G.N., Groumpos, P.P.: Brain tumor characterization using the soft computing technique of fuzzy cognitive maps. Applied Soft Computing 8, 820-828 (2008)

9. Groumpos, P.P., Stylios, C.D.: Modeling supervisory control systems using fuzzy cognitive maps. Chaos Solit. Fract. 11, 329-336 (2000)

10. Saade, J.J.: A unifying approach to defuzzification and comparison of the outputs of fuzzy controller. IEEE Transactions on Fuzzy Systems 4(3), 227-237 (1996) 